\title{
Fubilee Editorial
}

\section{Infective endocarditis 1937-1987}

\author{
IAN R GRAY \\ From Walsgrave Hospital, Coventry
}

By 1937, infective endocarditis had long passed the milestones of pathological recognition, clinical identification, and bacteriological diagnosis. It was in the latter stages of being a progressively debilitating, incurably fatal disease that usually affected young people. Many of them were admitted repeatedly to hospital for treatment which was depressingly ineffective. I remember seeing such patients as a student and house physician, and how helpless one felt in the face of this unrelenting condition. Antibacterial treatment with sulphonamides was pointing the way towards eradicating infections of this kind but it rarely helped in endocarditis.

When penicillin became available towards the end of the second world war it was in very short supply. Indeed, in those early days, it used to be extracted from the urine of treated patients so that it could be re-administered. The dosage used was small by today's standards and treatment often had to be curtailed because supplies were so scarce. The earliest results of treating infective endocarditis were disappointing, ${ }^{1}$ and it was not until 1944 that Loewe et al reported the recovery of seven patients given 200000 units of penicillin daily with heparin. ${ }^{2}$ As penicillin became more freely obtainable it was possible to treat large series of patients with infective endocarditis, and its efficacy was fully confirmed. ${ }^{3}$ In infective endocarditis caused by streptococci of the viridans type an adequate dosage of penicillin given for a sufficiently long time nearly always eradicated the infection. Other streptococci, such as Streptococcus faecalis, were often much less sensitive; however, the discovery of the antibacterial synergy between penicillin and streptomycin 4 extended the range of antibiotic treatment. Later on, other derivatives of penicillin were synthesised which had properties that were useful in the treatment of infective endocarditis. Ampicillin and more particularly, amoxycillin are much better absorbed than benzyl penicillin or the phenoxypenicillins when given by

Requests for reprints to Dr Ian R Gray, Walsgrave Hospital, Clifford Bridge Road, Walsgrave, Coventry CV2 2DX. mouth and they have a wider antibacterial spectrum. They were to make it possible to treat many patients with infective endocarditis with an oral antibiotic, so avoiding repeated injections or prolonged intravenous treatment. ${ }^{5}$

Some staphylococci are sensitive to penicillin and when such organisms caused infective endocarditis the disease could be treated in the same way as a streptococcal infection. Most strains, however, produce penicillinase, which renders them resistant to penicillin. Until semisynthetic penicillins resistant to penicillinase, such as methicillin, were developed such infections could seldom be overcome. ${ }^{6}$

Isoxazole penicillins, which combine resistance to penicillinase with resistance to gastric acid, made oral antistaphylococcal treatment possible. As the penicillins became more versatile, other antibiotics appeared that had comparable bactericidal properties. Vancomycin, clindamycin, fucidic acid, rifampicin, and the aminoglycosides all have a role in the treatment of certain patients with infective endocarditis; but none of them has displaced penicillin as the antibiotic of first choice.

The advent of penicillin and the antibiotic revolution which followed made it possible to eradicate the infection in nearly all cases of infective endocarditis, provided the diagnosis was made and treatment initiated sufficiently early. This unquestionably was the most dramatic change in infective endocarditis in the past 50 years. Deaths from infective endocarditis in England and Wales fell from 1200 per annum in the 1930 s, when the disease was invariably fatal, to about 300 in 1950 . In the 1980 s there are still between 200 and 300 deaths each year (Registrar General's Statistics, personal communication). If one assumes a case mortality of 20 per cent there must still be an annual incidence of more than 1000 (more than 20 cases per million population). The incidence of infective endocarditis is about the same as it was in pre-antibiotic times.

The disease itself has changed in several ways. One outstanding difference is in the age of patients in whom infective endocarditis occurs. In the $1930 \mathrm{~s}$ 
nearly two thirds of them were less than 40 years old. The age of patients has risen steadily so that infective endocarditis is now a disease of the middle aged and elderly. ${ }^{7}$ The main reason for this is the decline in rheumatic fever-nowadays few young people have rheumatic valve lesions, which are vulnerable to infection. The routine closure of patent ductus has also subtracted a considerable number of young people from those at risk. Improved standards of dental hygiene in children may well have lessened the risk of infection from this source. At the same time, the longer survival of an earlier generation of those with rheumatic heart disease, some of whom have undergone valve replacement, provides an aging population that is susceptible to infection.

Whereas there are fewer infections of "rheumatic" mitral valves in young people, we now recognise that mitral valve prolapse is an important predisposing condition; and in older subjects degenerative changes in the mitral and aortic valves often seem to be important sites of infection. We have also come to realise that in many patients with infective endocarditis an affected valve was previously normal. A large and increasing number of patients have undergone valve replacement. Prosthetic valves have proved to be more vulnerable to infective endocarditis than the natural valves they replace. When prosthetic valve endocarditis occurs shortly after the operation it is usually a staphylococcal infection and mortality rates are high. An infection which occurs more than two months after operation (late prosthetic valve endocarditis) tends to be caused by the same organisms that infect natural valves. ${ }^{8}$ Such infection can usually be controlled, but there is the risk, peculiar to mechanical valves, of the prosthesis becoming obstructed and immobilised by vegetations. Cardiac surgery is not the only intervention that can be responsible for extraneous infection of a heart valve. Infection may follow haemodialysis for renal failure; it can happen after pacemaker implantation and in patients on prolonged intravenous medication. Infective endocarditis also affects drug addicts who use contaminated needles and syringes for intravenous injections. ${ }^{9}$

Despite the various kinds of extraneous infection which have appeared in recent years, and which tend to be staphylococcal, there has been surprisingly little change in the spectrum of bacteria that cause infective endocarditis. Streptococci, mainly of the viridans type, continue to be responsible for between 60 and 70 per cent of infections, with staphylococci causing 20 to 30 per cent. Staphylococcus epidermidis has emerged as an important cause of infective endocarditis, particularly in prosthetic valve infections; Coxiella burnetii endocarditis is now well recognised, and fungal infection, usually with Can- dida, is a rare but serious problem.

The first intimation that surgery might have a part ${ }^{c} \cdot$ to play in the treatment of infective endocarditis was in 1940 when Touroff and Vessell reported the re- $\frac{-}{0}$ covery of a patient with infective endocarditis after ligation of an infected ductus. ${ }^{10}$ The major indi- $-\overline{\bar{\omega}}$. cations for surgery could not be realised, however,, until valve replacement became possible. Before an- $\frac{2}{0}$ tibiotics were available most patients with infective $e^{\infty}$ endocarditis died of uncontrolled infection. A re- $\overrightarrow{0}$ view of deaths from infective endocarditis in the $\overrightarrow{-}$ 1950s revealed that by then cardiac failure had be- $\omega$ come the principal cause of death. ${ }^{11}$ Cardiac failure in infective endocarditis is often caused by damage to valve structures by the infective process. There. can be perforation of a valve cusp, detachment of $a n_{i}$ aortic cusp from the wall of the aorta, or rupture of chordae of the mitral valve. These lead to acute valveo regurgitation which can cause a devastating volume overload of a small non-compliant recipient cham- $z$ ber with the rapid onset of severe left heart failure.

When valve replacement began to be widely prac- tised in the early 1960s, acute valve rupture in infec- $\overrightarrow{0}$ tive endocarditis was perceived to be an importan indication. Because of the dangers of carrying out major cardiac surgery in an infected field, valve re-O placement was at first postponed until antibiotic treatment had been completed. It soon became ap-ô parent, however, that the benefits of correcting then acute valve lesion outweighed such potential dan- $\frac{0}{\vec{t}}$ gers, and valve replacement was increasingly carried, out before the infection was fully controlled. ${ }^{12}$ The $^{3}$ improvement that often followed the operation was more dramatic and immediate than was usually the case in valve replacement for longstanding mitral or aortic regurgitation, and after 20 years it can still be seen in retrospect to have been a lifesaving inter-0. vention. With the success of valve replacement in patients with acute valve regurgitation even whiles infection was still active, it was a short step to valve replacement for the treatment of antibiotic resistane infections, covering the procedure with the bes $\$$ available antimicrobial regimen. Valve replacemente. is also necessary when prosthetic valve endocarditis causes obstruction of a mechanical prosthesis and in some patients who have had a major systemic em bolus; it is even considered in patients with evidences of extensive vegetations.

Echocardiography is useful in infective endoo carditis. $M$ mode echocardiography, and, morø particularly, cross sectional echocardiography provide a means of identifying larger vegetations on the heart valves, and also can demonstrate, withou of recourse to invasive forms of investigation, serious valve damage requiring surgical treatment. Echo@ cardiography has its limitations: failure to demon? 
strate vegetations does not exclude the diagnosis of infective endocarditis, and the presence of vegetations does not necessarily imply that the disease is still active. It can sometimes be difficult to distinguish vegetations from a thickened or calcified valve, and prosthetic valves are very difficult to investigate. The technique, however, plays an important part in assessing vegetations, in following the progress of the disease, and in assessing the need for surgical intervention.

At the same time as antibiotic treatment of infective endocarditis became possible, antibiotic prophylaxis covering dental and other interventions became widely recommended-recommended but not necessarily practised. The facts that the incidence of infective endocarditis has hardly changed and that unprotected dental procedures and poor dental hygiene are still responsible for many cases of infective endocarditis speak for themselves. ${ }^{13}$ The introduction of adequate oral rather than intramuscular antibiotic cover should increase the proportion of protected dental procedures, ${ }^{14}$ but unfortunately in many of those in whom infective endocarditis develops heart disease is not recognised before the procedure, and prophylaxis cannot be considered. It is possible, too, that in such a common condition as mitral valve prolapse, the risk of infection may be outweighed by the risk of penicillin prophylaxis. When adequate antibiotic prophylaxis is given infective endocarditis rarely occurs. There are still many unanswered questions as to who requires antibiotic prophylaxis and in what circumstances, and we still do not know the portal of entry of infection in most cases of infective endocarditis.

The continuing mortality from infective endocarditis remains both a challenge and a reproach. ${ }^{15}$ It is a challenge because we now have the means of treating effectively the microbial infections responsible for most cases and of correcting the destructive effects of infection on the heart valves. Despite this, antibiotic treatment is sometimes inadequate or inappropriate, and the need for surgical intervention is often unrecognised. It is a reproach because many cases of infective endocarditis are still only recognised at necropsy, and in many others the diagnosis is made only after weeks of illness. Delay in diagnosis means that a patient is at risk of major systemic embolism, cardiac failure, or renal damage for longer than need be. To fail to make the diagnosis of infective endocarditis in its early stages in 1937 was unfortunate: in 1987 it could be a disaster.

\section{References}

1 Florey ME, Florey HW. General and local administration of penicillin. Lancet 1943;i:387-97.

2 Loewe L, Rosenblatt P, Greene HJ, Russell M. Combined penicillin and heparin therapy of subacute bacterial endocarditis: report of seven consecutive successfully treated patients. $\mathcal{F} A M A$ 1944;124: 144-9.

3 Christie RV. Penicillin in subacute bacterial endocarditis. Report to the MRC. Br Med f 1948;i:1-4.

4 Hunter TH. Use of streptomycin in treatment of bacterial endocarditis. Am F Med 1947;2:436-42.

5 Gray IR. The choice of antibiotic for treating infective endocarditis. $Q \mathcal{F}$ Med 1975;44:449-58.

6 Rolinson GN, Stevens S, Batchelor FR, Wood JC, Chain EB. Bacterial studies on a new penicillinBRL1241. Lancet 1960;ii:564-7.

7 Hayward GW. Infective endocarditis: a changing disease. $\mathrm{Br} \mathrm{Med} \mathcal{F}$ 1973;ii:706-9; 764-6.

8 Slaughter L, Morris JE, Starr A. Prosthetic valvular endocarditis. A 12 year review. Circulation 1973; 47:1319-26.

9 Dreyer NP, Fields BN. Heroin-associated infective endocarditis. A report of 28 cases. Ann Intern Med 1973;78:699-702.

10 Touroff ASW, Vessell H. Subacute streptococcus viridans endarteritis complicating patent ductus arteriosus: recovery following surgical treatment. JAMA 1940;115:1270-2.

11 Robinson MJ, Ruedy J. Sequelae of bacterial endocarditis. Am $\mathcal{F}$ Med 1962;32:922-8.

12 Yeh TJ, Hall DP, Ellison RG. Surgical treatment of aortic valve perforation due to bacterial endocarditis: a report of 6 cases. Am Surg 1964;30:766-9.

13 Bayliss R, Clarke C, Oakley C, Somerville W, Whitfield AGW. The teeth and infective endocarditis. Br Heart $\mathcal{f} 1983 ; 50$ :506-12.

14 Working Party of the British Society for Antimicrobial Chemotherapy. The antibiotic prophylaxis of infective endocarditis. Lancet 1982;ii:1323-6.

15 Goodwin JF. The challenge and the reproach of infective endocarditis. $\mathrm{Br}$ Heart $\mathcal{F}$ 1985;54:115-8. 